\title{
Conceptual Designing of Energy Efficient Optimal Path Routing Technique for Ad-hoc Network using Genetic Approach
}

\author{
Meenu Vijarania \\ Assistant Professor \\ Amity University, Haryana
}

\author{
Vivek Jaglan, PhD \\ Assistant Professor \\ Amity University, Haryana
}

\author{
Sonam Rani \\ M.Tech student \\ Amity University, Haryana
}

\begin{abstract}
Power aware routing is the effective way to increase the life span of nodes having limited battery power in ad hoc networks. In MANET, Energy consumption is a significant issue since all of the mobile nodes are battery powered due to which lifetime of a network is restricted. In order to prolong the lifetime of ad hoc networks, the energy consumption of nodes should be minimized sincerely, exhaustion of energy of a single node causes link breakage in a network. In this paper, energy conscious routing procedures are analysed and an algorithm is proposed with simulation in MATLAB that will help to enhance the lifetime of network. Genetic algorithm gives the random solutions from the generations of paths and achieves the optimized performance. From the tentative consequences, it is resolved that network will be more energy efficient as compared to the earlier energy efficient algorithms.
\end{abstract}

\section{Keywords}

MANET, energy aware routing, genetic algorithm

\section{INTRODUCTION}

Mobile Ad-hoc Networks (MANETs) are composed of a number of mobile nodes which can move freely in an arbitrary manner without of any fixed infrastructure. Due to mobility of nodes, the topology of the network fluctuatesenthusiastically. To transfer a message or packet, any number of nodes can participate in a communication.

The Energy Efficient Routing protocols always try to use the more efficient path that consumes less of energy since all of the mobile nodes are limited battery powered in a network. When comparing theMANET with stablenetwork structure, the special features like dynamic topology, absence of any fixed links, limited battery capacity make it different and more suitable. The main tasks involved in MANETs are neighbour discovery, topology organization, and topology restructuring. The lifespan of a link is mainly based on battery power of each node on that link in a network. The early collapse of a single node affects the lifetime of complete network or may be the network will partitioned. The consumption of each node varies according to their state: transmitting, receiving, idle, listening or sleeping. These batteries cannot be easily recharged and replaced in a complex environment that's why nodes should be managed efficiently to prolong the lifetime of a network.

\section{LITERATURE SURVEY}

The energy aware routing protocols that are mainly used are MTPR(Minimum Total Transmission Power Routing), MBCR(Minimum Battery Cost Routing), MMBCR(Min.-Max Battery Cost Routing), CMMBCR(Conditional Min-Max
Battery Cost Routing), MDR(Minimum Drain Rate), CMDR(Conditional MDR).

The protocol named "MTPR" has introduced that only taken into account a single metric 'total transmission power' as the cost metric[3]. This protocol selects a path which consumes least transmission power to route a packet from source to destination. The transmission power is directly relational to transmitting distance from source to destination.

$$
\mathrm{T}_{\mathrm{p}} \alpha \mathrm{T}_{\mathrm{d}}\left(\mathrm{i}_{0}, \mathrm{i}_{\mathrm{n}}\right)
$$

$\mathrm{T}_{\mathrm{p}}-$ transmitting Power

$\mathrm{T}_{\mathrm{d}}$ - transmitting Distance

n- total no. of nodes

$\mathrm{i}_{0-}$ source node

$\mathrm{i}_{\mathrm{n}}$ - destination node

This protocol may select the path which is having more no. of hops due to which there will be longer delay to passing a message. The lifetime of a path will be less because chances to break a path will be higher. It also not considers residual battery power of a node. If on a selected path, a single node has no any residual battery power then total network will be end up. The total transmission power not only considers power consumed for transmitting it also consider for receiving a packet also[12].

The authors proposed a new protocol named 'MBCR'[1]. This protocol has resolved the problem of not considering the residual battery power in a route. Although the protocol MTPR considers minimum of total transmission power consumption of overall network but it does not directly affect the lifetime of nodes. The MBCR protocol considers a metric remaining battery power as the cost function[15]. The path which has maximum remaining battery capacity is selected. Battery capacity is inversely proportional to cost function calculated on that path. If battery capability of a network is ina smaller amount, then the cost function value will be more.

Cost function $\left[\mathrm{f}\left(\mathrm{C}_{\mathrm{n}}\right)\right]=1 / \operatorname{Capacity}\left(\mathrm{C}_{\mathrm{n}}\right)$

The the path of maximum remaining battery capacity is selected but the problem is that here the nodes in a path may be worn-out.

The problem occurred in MBCR protocol has resolved that it only considers sum of residual battery power of a path not of individual nodes[1]. It may be possible that the sum is greater but a single node has less RBP due to which overall path in a network will exhaust.

This protocol guarantees that no node will be overworked. It firstly drafts the residual battery power of all his neighbours and the host containing maximum RBP is selected. This process is going on until the complete path from source to 
destination has been found out. When comparing MBCR with MMBCR the lifetime of a path will be longer in MMBCR[2]. But the common problem of these protocols is that they do not reflect the metric "total transmission power". So they can elect the lane whichconsumes a huge amount of transmission power.

CMMBCR protocol has resolved the problem of MMBCR protocol[4]. This protocol uses a fusionmethodology of MTPR and MMBCR[17]. It chooses the path which consumes less of total transmission power and also concentrates on remaining battery capacity of individual nodes belonging to a path in a network. If there are a large no. of path that contains sufficient remaining battery capacity then the path which consumes less of transmission power is selected and lifetime of a network is prolonged. By setting the threshold value in a network, the nodes can easily be protected from early depletion/exhaustion. The energy efficient routing protocols can't give the best results only on the basis of remaining battery capacity. To prolong the lifespan of nodes, the links having little battery size are always avoided. There may be a possibility that at any time the node is willing to accept all the RREQ packets but at the same time the traffic injected to that particular node will be very high. In this sense, actual drain rate of power consumption will be high that results to sharp exhaustion of battery power.

The author proposed a new protocol named MDR in which he used a new and different metric named 'Drain rate'[6]. Drain rate mainly concentrates on current traffic condition. This protocol takes into account the drain rate index and residual battery capacity to calculate the cost function. Cost function measures the energy dissipation rate of a node in a network. The value of cost function should be minimum. Drain rate calculates the average energy consumed per second at a node. It will always select the path which has maximum lifetime. The path of minimum $c_{i}$ will having maximum lifetime among all the available paths.

This protocol concentrates to extent the lifetime of nodes as well as of connection, but here is a problem that it does not taken into account the metric 'total transmission power'. The selected path may consume a large amount of transmission power. It also not confirms the least energy budgetpathway.

The protocol named CMDR has resolved the problem of MDR protocol[8]. This protocol also uses a fusionmethodology of MDR and MTPR[17]. It always chooses the path which is having minimum consumption of transmission power and longer lifetime as compared to all other available paths to transmit a packet. If no any route does not fulfil this condition then they have to switch the MDR mechanism. The threshold value can be taken into account by concentrating the current traffic condition in a network. It also represents how long a path will be stable with any of the energy breakage.

\section{PROBLEM FORMULATION}

The node in MANET mainly operates on battery power since they have limited battery capacity. Due to limited battery, the nodes in a network will easily be deceased and the network will be partitioned. As well as the lifetime of the network will be restricted. The partitioning of network due to excursion of battery can be handled by routing decisions.Here, the main concentration is on how the lifetime of network can be prolonged.There is a big issue of link damage which mainly occurs due to the dynamic topology of network or exhaustion of a node in network. If a link is damaged and it is of no future use then a message is send to all other nodes that will use this node in their communication. Then there will be a large delay for forwarding a packet by avoiding the broken link. The problem of link failure and overhead occurred due to these links failure require more attention. AODV protocol is flexible to highly dynamic network but they have more delays due to reconstruction of path re-establishment or re-discovery of path. DSR is advantageous over AODV protocol because of presence of route cache in which it stores the multiple paths from source to destination but it is not scalable to large networks and requires more resources. So AODV protocol performances batter as compared to others,

There is an option to save the data on broken links is that to use of backup paths. With the help of backup paths, the data can be saved if the route is destroyed due to link breakage[11] But backup route may have changed due to topological changes so main concentration is on that the topological changes will not be reflected in backup routes.

For balancing the power consumption load balancing techniques can be applied to improve the network performance [14]. It also reduces end to end delay and robustness of data delivery. These techniques evenly distribute the traffic which helps in efficient energy utilization that directly affect the network lifetime.

Load balancing can be done by single path routing or multipath routing.

\section{PROPOSED ALGORITHM}

1. Initialize the network or initialize the chromosomes. Identify source and destination nodes. Then check for all the available paths between source and destination.

2. Calculate the fitness value according to the metrics applied.

i. Firstly, the total transmission power is checked to select the path which consumes least energy for transmission.

ii. The remaining battery power is calculated from initial battery power they had and which of amount they have already used.

$$
\mathrm{Rbp}=\mathrm{IE}-\mathrm{TE}
$$

iii. Then check for the threshold value $(\gamma)$ of all the nodes. Threshold value is set by user initially in a network to maintain the stability.

$$
\mathrm{Rbp}>=\gamma
$$

iv. The drain rate index is calculated by their previous value.

$$
\text { DRi }=\alpha \times \text { DRold }+(1-\alpha) \times \text { DRsample }
$$

3. After that by using the drain rate index and RBP the cost function calculated is

$$
\mathrm{C}_{\mathrm{i}}=\mathrm{RBP}_{\mathrm{i}} / \mathrm{DR}_{\mathrm{i}}
$$

4. Value of cost function should be minimum to maximize the lifetime of network nodes as well as of connection.It selects a route with nodes having maximum cost function value among the minimum cost functions of a path.

$$
\begin{aligned}
& \operatorname{Min}\left(C_{i}\right)=\max . L_{P} \\
& L_{p} \text { - lifetime of path }
\end{aligned}
$$


5. After checking these all of the metrics a path can be established from source to destination.

6. But at a time if a large traffic is injected on a node in a route that node will exhaust or link will be broken. So randomly it can choose one another path among the feasible paths.

7. Apply GA operations through GA tool in MATLAB

$$
\begin{array}{ll}
\text { * Selection } \\
\text { * Crossover } \\
\text { Mutation }
\end{array}
$$

\section{RESULTS AND SIMULATION}

Efficient energy utilisation and balancing of nodes cannot be easily achieved. Here, the performance of the protocols can be evaluated through the metrics transmission power(MTPR), remaining battery power(MBCR) and drain rate(MDR) using the Matlab simulator with genetic algorithm. Genetic Algorithm is an optimization tool that helps to achieve the optimized solution for a given problem. Here the ad-hoc network is simulated with 6 nodes for the random number of generations. All nodes are designed as: Node-number (initial-energy, transmission-energy, drain rate)

Node $1=(65,5,2)$

Node $2=(80,10,3)$

Node $3=(20,10,4)$

Node $4=(95,15,5)$

Node $5=(35,20,6)$

Node $6=(120,10,4)$

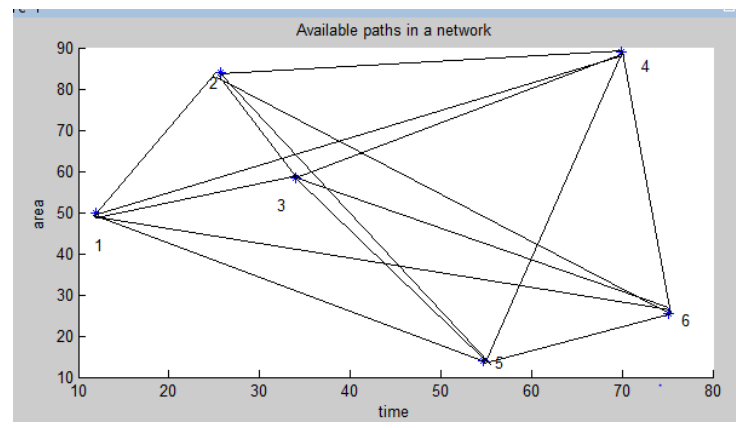

Fig 1: Example of MANET using 6 mobile nodes

The fitness value is calculated of all the available paths by applying the metrics through the MATLAB.

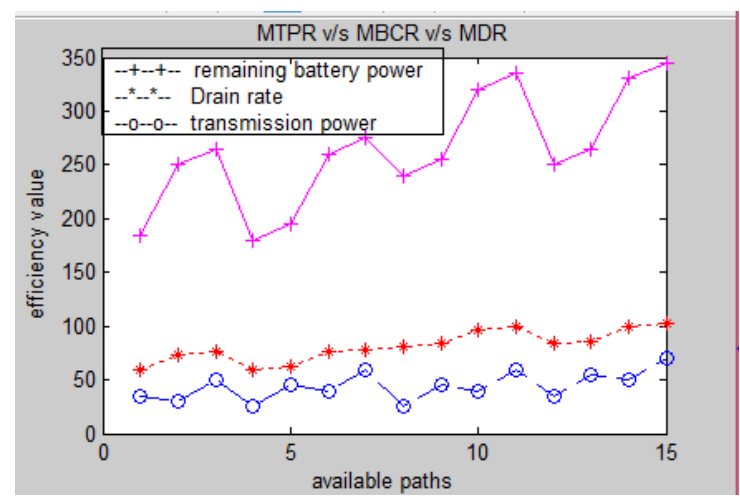

Fig 2: fitness value of all the paths
During route discovery, always try to select the best optimal route among all the available routes from source to destination. If applying the transmission power then it always selects the path which consumes least of transmission energy. But the path will not be the feasible path because here the traffic injected will be in large amount due to which there will be large delay for routing of message or data. Battery power is also consumed by node in a network in idle state too.

When applying remaining battery power then the path which is of having large remaining battery capacity is selected.But remaining battery power should be greater than threshold $(\gamma=$ 16)value set for each node. There is a chance that it can select the node in a route which has least battery capacity because it considers the sum of remaining battery power and the lifetime of path will be restricted. This path will not be continued for a longer time because the link to that node will be broken or ultimately the network will be partitioned which cause interruption of service in a network. After checking the threshold value and remaining battery power of nodes the feasible paths are

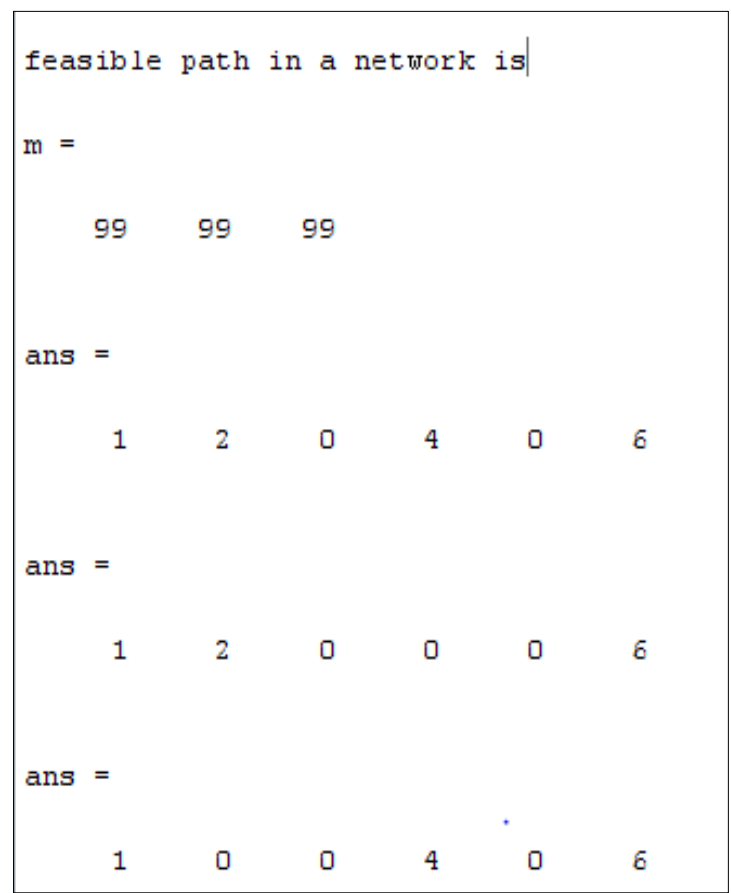

Fig 3: feasible path calculated through MATLAB

The drain rate mainly concentrates on current traffic conditions. It processes the data by checking the all node's drain rate index and remaining battery power. The lifetime of a path is checked by calculating the cost function $\left(\mathrm{C}_{\mathrm{i}}\right)$ through remaining battery power and drain rate index:

$$
\mathrm{C}_{\mathrm{i}}=\mathrm{RBP}_{\mathrm{i}} \mid \mathrm{DR}_{\mathrm{i}}
$$

The minimum value of cost function of a node is considered as the lifetime of a path as well as of connections. It selects a route with nodes having maximum cost function value among the minimum cost functions of a path. In a path, which node has maximum cost function value $\left(\mathrm{c}_{\mathrm{i}}\right)$ that path will be used only for same no. of times.

$$
\mathrm{L}_{\mathrm{p}}=\min \left(\mathrm{C}_{\mathrm{i}}\right) \text { of a path }
$$

After applying the metrics the feasible paths from all the available paths are calculate in MATLAB. 


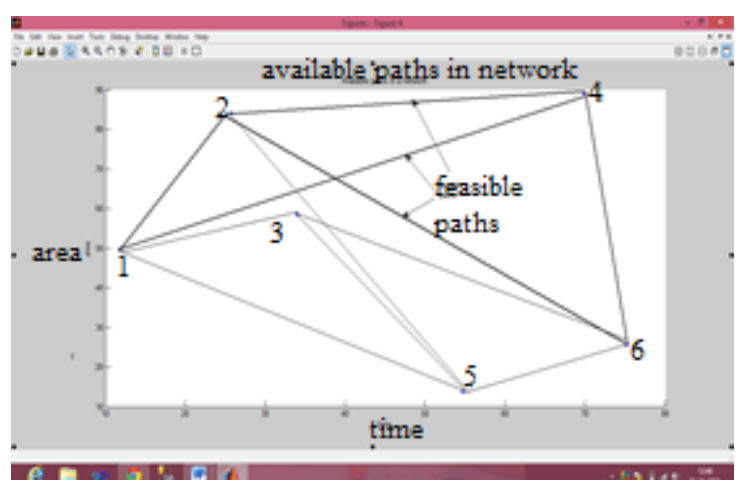

Fig 4: feasible paths in MANET

$$
\begin{aligned}
& \text { Min. } C_{i} \text { of path }(p 1)=16 \\
& \text { Min. } C_{i} \text { of path }(p 2)=23 \\
& \text { Min. } C_{i} \text { of path(p3) }=16
\end{aligned}
$$

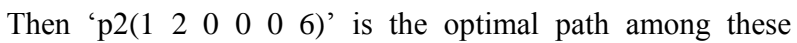
feasible paths.

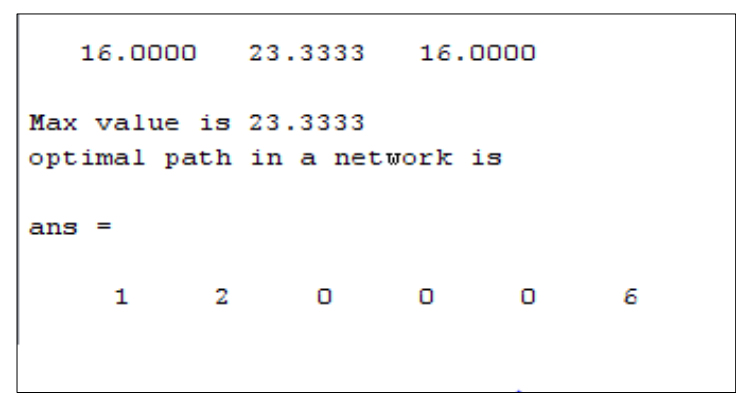

\section{Fig 5: Optimal path through MATLAB}

Then the optimal path in a network is chosen by checking their fitness value through GA tool and applying the genetic algorithm operations.

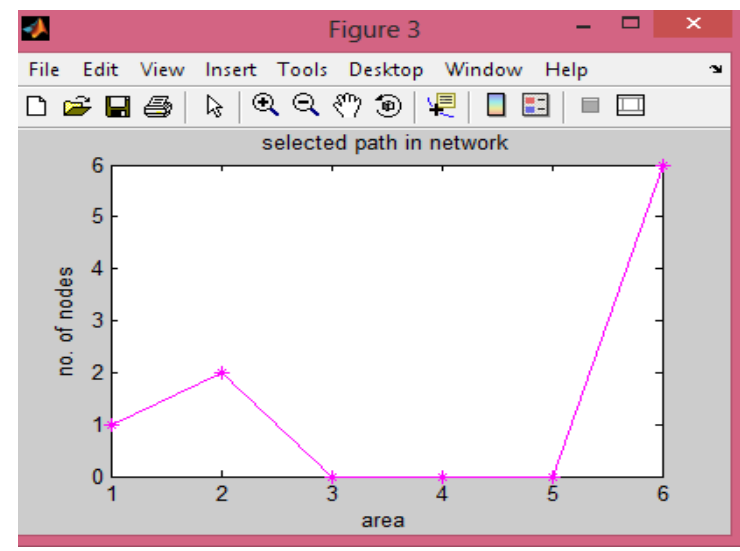

Fig 6: optimal path in a network(graphically)

The path is selected is of higher fitness value. After calculating the fitness value of all the feasible paths. And then selection operation is selected in GA tool by the given functions. Crossover and mutation rate is also applied. The generation is set as bit string and give the generation from generation function which is created.
The fitness value is calculated of all the nodes in feasible paths through the GA tool by applying random no. of iterations.

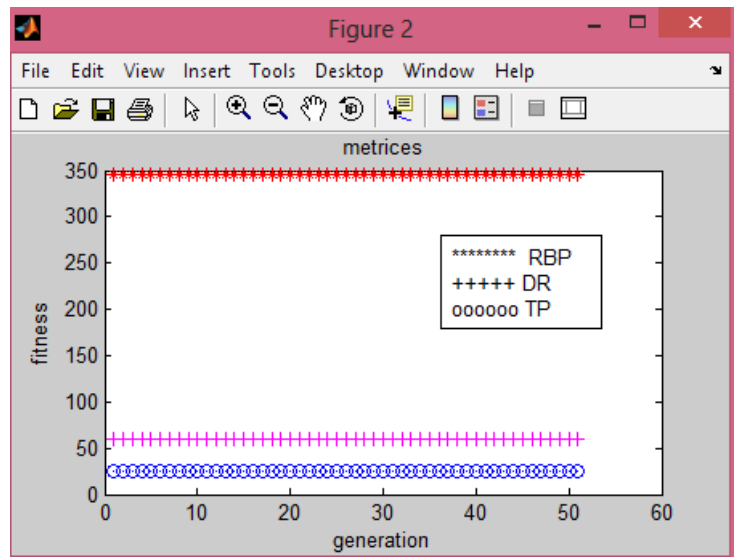

Fig 7: fitness calculated for metrics through GA tool

The lifetime of nodes in a network can also be found out which mainly shows that how many nodes are alive up to which time and which no. of nodes are dead because dead nodes do not participate in the communication in a network.

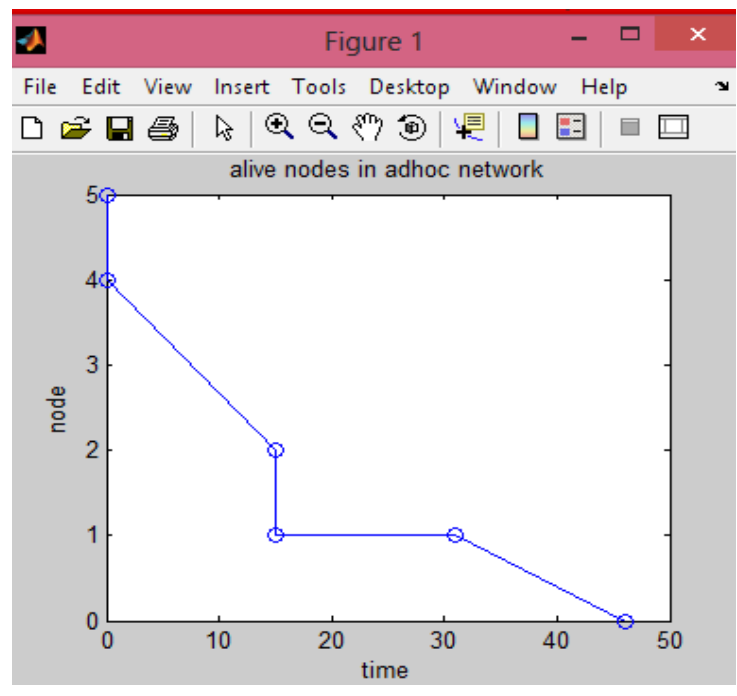

Fig 8: alive nodes in network

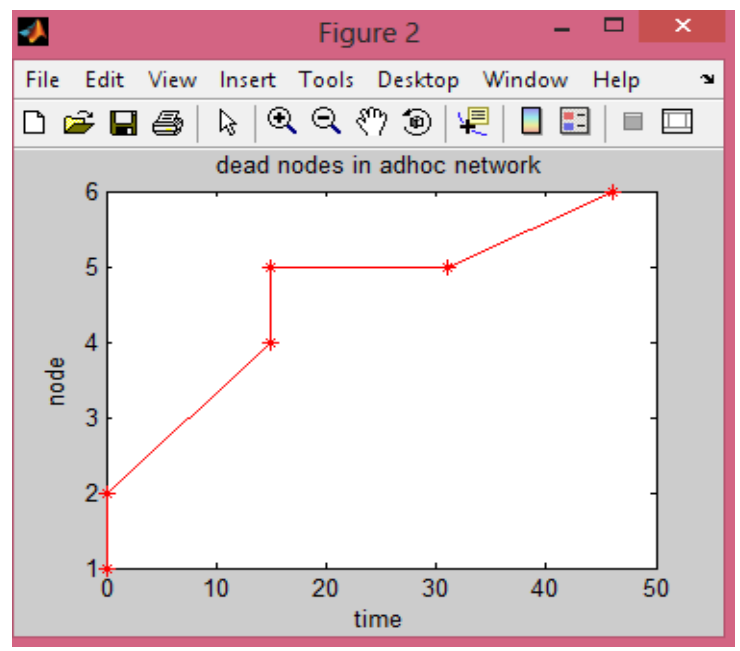

Fig 9: dead nodes in a network 


\section{CONCLUSION}

In the paper, all of the available paths with their connection probability are generated. Firstly, paths are checked according to their threshold value. A node having less than threshold will not be included in a path. After that the fitness value is checked of all the feasible paths by applying the metrics. The path with higher fitness value is always chosen because higher fitness valued chromosomes will be more suitable for solving the problem. Then results are then calculated with the help of GA tool in MATLAB which shows GA gives better results with random number of generations. The GAs always provide robust and efficient search in complex spaces and are used to solve an optimization problem. In future work, GA with MATLAB can also be used to obtain comparatively better results and can be implemented for other process controllers. In MANET, the research using genetic approach is an emergent field.

\section{REFERENCES}

[1] S:J. Lee, M. Gerla, and C.-K. Toh, "A Simulation Study of Table-Driven and On-Demand Routing Protocols for Mobile Ad Hoc Networks," I€€€ Network, July 1999

[2] E. Royer and C.-K. Toh, "A Review of Current Routing Protocols for Ad Hoc Mobile Wireless Networks," /€E€ Pen Commun..vol. 6, no. 2, Apr. 1999, pp. 46-55.

[3] Madhavi W. Subbarao, Member, IEEE(1999) "Dynamic Power-Conscious Routing for MANETs: An Initial Approach" IN National Institute of Standards and Technology, Gaithersburg, MD, USA .

[4] C.-K. Toh(2001), "Maximum battery life routing to support ubiquitous mobile computing in wireless ad hoc networks," IEEE Communications Magazine, June 2001.

[5] W. Cho and S.-L.Kim(2002), "A fully distributed routing algorithm for maximizing lifetime of a wireless ad hoc network" in 4th International Workshop on Mobile and Wireless Communications Network.

[6] Dongkyun Kim, J.J Garcia-Luna-Aceves and Katia Obraczka, Juan-Carlos Cano and Pietro Manzoni(2002) "Power-Aware Routing Based on The Energy Drain Rate for Mobile Ad Hoc Networks"

[7] M. Maleki, K. Dantu, and M.Pedram(2002) "Power-aware source routing protocol for mobile ad hoc networks," in ISLPED.

[8] S. SankaraGomathi, S. Krishnamurthi, Richard Chbeir(2003) "Design of Extended Optimal Energy
Drain Rate Algorithm for Mobile Ad-hoc Network" from department of computer science and electronics and communication from Sri Venkateswara College of Engineering and Anna University

[9] Lijuancao, Teresa Dahiberg, Yu Wang(2007) "performance Evaluation of Energy efficient adhoc routing protocols" in University of North Carolina at Charlotte, Deptt. Of computer science.

[10] Dilip Kumar S.M. andVijaya Kumar B.p.(2008) "An Efficient Multicast Routing in MANETs: A Genetic Algorithm approach" in International Journal of Information and Computation technology.

[11] Ashish Kumar, M. Q. Rafiq, Kamal Bansal(2011) “A Survey of Link Failure Mechanism and Overhead of Routing Protocols in MANET" in (IJCSIT) International Journal of Computer Science and Information Technologies, Vol. 2 (5).

[12] DharamVir, S.K.Agarwal, S.A.Imam and LalitMohan4(2012) "PERFORMANCE ANALYSIS OF MTPR ROUTING PROTOCOL IN POWER DEFICIENT NODE"at Department of Electronics Engineering, YMCA University, Faridabad, India

[13] Sonam Jain, SandeepSahu(2012) "The Application of Genetic Algorithm in the design of Routing Protocols in MANETs: A Survey" from Computer Science \& EngineeringShri Ram Institute of Technology Jabalpur, India.

[14] DepallySubashSudheer,Dr. RameswarBaliarsingh (2013) "Load Balancing in MANET : Alleviating the center node" in National Institute of Technology Rourkela.

[15] V.Seethalakshmi ,Dr. G. Mohan Kumar (2013)“A Survey of Energy Aware Ad Hoc Routing Protocol" at International Journal of Emerging Technologies in Computational and Applied Sciences (IJETCAS).

[16] AnjumAsma(2013) "Energy Efficient Routing Algorithm for Maximizing Network Lifetime of MANETs" Dept. of I.T., CCIS, King Saud University, Riyadh, Kingdom of Saudi Arabia in International Journal of Innovative Research in Computer and Communication Engineering.

[17] MeenuVijarania ,VivekJaglan, "Comparative Analysis of Various Energy Efficient Routing Protocols in Adhoc Networks", International Journal of Recent Research Aspects ISSN: 2349-7688, Vol. 2, Issue 1, March 2015, pp. 60-62. 